\title{
EFFECTS OF SOMATOSTATIN ANALOGUE (OCTREOTIDE) ON ISCHEMIA-REPERFUSION INJURY IN ADULT HYPERTHYROID ALBINO RATS
}

\author{
By \\ Wessam Ezzat Morsy \\ Department of Medical Physiology, Faculty of Medicine, Ain Shams University \\ Corresponding author: doctorapioneera@yahoo.com
}

\begin{abstract}
Background: Thyroid dysfunction is associated with increased cardiovascular morbidity and mortality. Hyperthyroidism increases the susceptibility of the heart to ischemia reperfusion injury. Moreover, myocardial ischemia can be further worsened by increased cardiac workload and oxygen demand elicited by the excess thyroid hormones.

Objectives: Investigating the cardiac dysfunction induced by ischemia-reperfusion (IR) in rats exposed to excess thyroid hormones and role of somatostatin analogue against ischemia/reperfusion injury in hyperthyroid rats.

Patients and Methods: This study was performed on 24 adult male albino rats of a local strain weighing 140-200 g, allocated into three equal groups: Group I (Control IR group): Euthyroid control group exposed to ischemia-reperfusion. Hearts of this group were isolated and perfused in a Langendorff apparatus. Isolated hearts were subjected to $15 \mathrm{~min}$ of stabilization, $20 \mathrm{~min}$ of zero-flow global ischemia and $30 \mathrm{~min}$ of reperfusion. Group II (Hyperthyroid IR group) were subjected to the same protocol of ischemia reperfusion. Group III (Hyperthyroid IR-OCT group): Hyperthyroid rats were supplemented with the somatostatin analogue, octreotide (OCT), $35 \mu \mathrm{g} / \mathrm{kg}$ subcutaneously, 20 minutes before sacrifice, followed by in-vitro study of cardiac responses to ischemia reperfusion. Blood samples were collected for measurement of plasma free T3, T4 and TSH. Coronary efflux lactate dehydrogenase levels were measured. Also, cardiac tissue malondialdehyde (MDA) levels and tumor necrosis factor alpha (TNF- $\alpha$ ) levels were estimated.
\end{abstract}

Results: Hyperthyroid (IR) rats revealed significant elevation in plasma free T3 and T4 with concomitant decrease in TSH levels. A significant reduction in final BW and BW gain were observed with higher absolute weights of left ventricle (LV) and cardiac index compared to the euthyroid controls. Systolic blood pressure (SBP) and mean arterial pressure (MAP) significantly elevated in non-treated hyperthyroid rats with a significant increase in their baseline chronotropic and inotropic activity compared to euthyroid control rats. But after ischemia those rats exhibited significantly higher degree of bradycardia $(\Delta \mathrm{HR})$ and $\%$ change in heart rate (HR) compared to control rats. Moreover, significantly deteriorated post-ischemic recovery of inotropic parameters and significantly higher $\Delta$ changes of these parameters were observed compared to euthyroid controls. These changes were accompanied by significant elevation in coronary efflux LDH levels and both cardiac tissue MDA and TNF $\alpha$. Meanwhile, octreotide administration to hyperthyroid rats showed improvement of their baseline chronotropic and inotropic parameters as evidenced by the significantly reduced degree of bradycardia as well as significantly lower $\Delta$ change of PT and PT/LV parameters compared to non-treated hyperthyroid rats. Such findings could be ascribed partly to the reduction of 


\section{WESSAM EZZAT MORSY}

oxidative and inflammatory responses to IR as determined by the significantly decreased cardiac tissue MDA and TNF $\alpha$ respectively.

Conclusion: Hyperthyroid hearts are less tolerant to ischemia/reperfusion injury with higher susceptibility to oxidative stress as well as overproduction of pro-inflammatory mediators. Thus, pharmacological targets that defend oxidative challenge and confer anti-inflammatory properties, namely octreotide (somatotstatin analogue) are considered to have potential therapeutic effect and may protect against myocardial ischemic damage in hyperthyroidism.

Key words: Ischemia reperfusion injury, hyperthyroid, oxidative stress, inflammation, somatostatin, octreotide.

\section{INTRODUCTION}

Thyroid hormones are known to play crucial roles in the regulation of cardiovascular functions. Cardiac inotropic, chronotropic properties as well as vascular resistance are directly affected by thyroid hormones levels (Seara et al., 2018).

Hyperthyroidism is associated with tachyarrhythmias, cardiac hypertrophy and heart failure with increased cardiovascular morbidity and mortality (Rodondi et al., 2008). The high levels of thyroid hormones increase the susceptibility of the heart to ischemia/reperfusion (IR) injury, elevate apoptotic rate of hypertrophied cardiomyocytes, and increase heart failure (Wang et al., 2010). Such deleterious effects are attributed to increased rate of ATP consumption, redox imbalance and overproduction of ROS encountered in hyperthyroidism (Zaman et al., 2014).

Moreover, myocardial ischemia can be further worsened by increased cardiac workload and oxygen demand elicited by the excess thyroid hormones (Pantos et al., 2011 and Seara et al., 2018).

Somatostatin (SS) is a peptide, known predominantly for its inhibitory action on growth hormone, however, SS is localized in the nerve fibers within muscle bundles of both the atria and ventricles with a surprising high level of somatostatin receptors (SSTR) expressed in the rat heart with different distribution of the receptor subtypes in cardiac fibroblasts and cardiomyocytes (Widiapradja et al., 2017).

Octreotide, a synthetic analogue of somatostatin can mimic ischemic preconditioning (PC) to provide cardioprotection against myocardial infarction through activation of protein kinase $\mathrm{C}$, tyrosine kinase $\mathrm{C}$, and mitochondrial ATP-sensitive potassium channels (Wang et al., 2005).

Although thyrotoxic hearts are more vulnerable to IR damage, up to our knowledge, only a recent study performed by Seara et al. (2018) has issued the progression of ischemia reperfusion injury in rat hearts exposed to supraphysiological concentration of thyroid hormones .

In addition, most experimental data on somatostatin analogue has focused on the "non-diseased" myocardium (Wang et al., 2005) or other ischemic organ damage such as hepatic ischemia reperfusion injury (Yang et al., 2014). Yet, no available studies in the literature have accessed its cardioprotective role against IR injury in the presence of disease conditions such as hyperthyroidism . 
The present study was designed to investigate the cardiac dysfunction induced by ischemia-reperfusion in rats exposed to excess thyroid hormones and through more light on the potential role of somatostatin analogue against ischemia/reperfusion injury in hyperthyroid rats.

\section{MATERIALS AND METHODS}

\section{Experimental animals:}

The present study was performed on 24 adult male albino rats of local strain weighing 140-200 g. Rats were purchased from the Egyptian Organization for Biological Products and Vaccines (VACSERA) and were kept in the Animal House, Physiology Department, Faculty of Medicine, Ain Shams University under standard conditions of boarding and feeding with free water access. Animals were kept in their cages, $(30 \mathrm{~cm}$ width $\mathrm{x}$ $50 \mathrm{~cm}$ length $\times 20 \mathrm{~cm}$ height, 4 rats/cage), well ventilated, and in normal day/night cycle. Ethical approval for the study was given by the Research Ethics Committee, Faculty of Medicine, Ain Shams University (FMASU, REC, Cairo, Egypt). The study period was 8 weeks during which rats were allocated into three equal groups:

\section{Group I (Control IR group): Euthyroid control group exposed to ischemia-reperfusion. Hearts of this group were isolated and perfused in a Langendorff preparation. Isolated hearts were subjected to $15 \mathrm{~min}$ of stabilization, $20 \mathrm{~min}$ of zero-flow global ischemia and $30 \mathrm{~min}$ of reperfusion.}

Group II (Hyperthyroid IR group): Hyperthyroid rats were subjected to the same protocol of ischemia reperfusion.

Group III (Hyperthyroid IR-OCT group): Hyperthyroid rats were supplemented with the somatostatin analogue, octreotide (OCT), $35 \mu \mathrm{g} / \mathrm{kg}$ subcutaneously, 20 minutes before sacrifice, followed by in-vitro study of cardiac responses to ischemia reperfusion.

\section{Induction of Hyperthyroidism:}

Rats in the experimental hyperthyroid groups (groups II\&III) were given intraperitoneal injection of L-thyroxine (Sigma chemicals Co., St. Louis, MO), in a dose of $10 \mu \mathrm{g} / 100 \mathrm{~g}$ body weight, daily for 8 weeks (Jiang et al., 2001). Lthyroxine was dissolved in a minimum amount of $\mathrm{NaOH}$, and then the concentration was adjusted to $2 \times 10-3 \mathrm{M}$ by dilution with normal saline solution (Komeda et al., 1977). Final pH was adjusted at 7 . Euthyroid control rats (group I) were injected intraperitoneally with an equivalent volume of saline diluted-NaOH solution (the solvent for Lthyoxine) daily for 8 weeks.

\section{Administration of Octreotide (Somatostatin Analogue):}

It was given to group III in the form of sandostatin purchased from Novartis Pharm Co., in a dose of $35 \mu \mathrm{g} / \mathrm{kg}$ subcutaneously, 20 minutes prior to IR (Wang et al., 2005).

Measurement of Arterial Blood
Pressure: Arterial blood pressure
(systolic, diastolic and mean) was
performed the day before scarification
using the non-invasive small animal tail
blood pressure system (NIBP200A,
Biopac systems Inc., USA).




\section{WESSAM EZZAT MORSY}

\section{Experimental Procedures:}

On the day of sacrification, overnight fasted rats were weighed, and then injected intraperitoneally with $5000 \mathrm{IU} / \mathrm{Kg}$ B.W heparin sodium (Nile Company, Egypt). Fifteen minutes later, the rats were anesthetized with intraperitoneal injection of thiopental sodium (Sandoz, Austria), in a dose of $40 \mathrm{mg} / \mathrm{kg}$ B.W. A midline abdominal incision was made to expose and cannulate the abdominal aorta. Blood samples were taken from the abdominal aorta and collected in heparinized plastic tube which was then centrifuged at 4000 rpm for 10 minutes to separate plasma. The plasma was then pipetted into clean storage tubes and stored at $-20 \circ \mathrm{C}$ for later determination of free T3, T4, TSH.

\section{Isolated Heart Study:}

Isolated hearts were immediately transferred to a constant pressure Langendorff apparatus. A baseline recording was obtained at a speed of $50 \mathrm{~mm} / \mathrm{sec}$ to determine the baseline beating rate (HR), peak tension (PT),time to peak tension (TPT) and half relaxation time ( $1 / 2 \mathrm{RT})$. Myocardial flow rate (MFR) was determined by collecting the fluid passing out of the heart in a glass beaker for 3 minutes (Ayobe and Tarazi, 1983).

Total global ischemia was induced by stopping of the perfusion fluid delivered to the heart by a clamp for 20 minutes. Cardiac responses (HR, PT, TPT\& $1 / 2 \mathrm{RT}$ ) and MFR were recorded during 5, 15 and 30 minutes of reperfusion following 20 minutes of total global ischemia. The values of different cardiac responses were calculated from the recordings as follows:
The heart rate (HR, beats per minute) was calculated by the formula:

HR: 3000 was divided by the distance in (mm) between two successive peaks of tension.

For irregular HR: 15000 was divided by the distance between 5 successive peaks.

The peak tension (PT, g), which is the amplitude of the recorded contraction force, was measured in $\mathrm{mm}$ and equivalent force in $\mathrm{g}$ was obtained from calibration curve. The peak tension was calculated per left ventricular weight (PT/LV, g/1b00mg).

The myocardial flow rate (MFR, $\mathbf{m l} / \mathbf{m i n}$ ) was calculated per minute. MFR was, also, calculated per left ventricular weight (MFR/LV, $\mathrm{ml} / \mathrm{min} / 100 \mathrm{mg}$ ).

\section{Determination of Cardiac Weights:}

Following heart perfusion, hearts were washed with normal saline, dried by filter paper and cleaned from fat and fibrous tissue. They were wrapped in parafilm and frozen at $-80 \circ \mathrm{C}$. Cardiac chamber weights (atria, right ventricle and left ventricle) and whole heart weight were expressed as absolute value in (mg) as well as relative values (absolute weight/ body weight ratio, cardiac indices) in $\mathrm{mg} / \mathrm{g}$.

\section{Biochemical Analysis:}

- Plasma Free T3, T4 and TSH Levels were carried out by electrochemiluminescence immunoassay "ECLIA" techniques, using kits supplied by Roche Diagnostics Switzerland (Fisher, 1996).

- Cardiac Tissue Malondialdehyde (MDA) Levels were measured according to the technique of (Ohkawa 
et al., 1979). This was performed using kits supplied by Bio-diagnostic, Egypt.

- Tumor Necrosis Factor Alpha (TNFa) Levels in Cardiac Tissue were determined via a commercial rat quantitive ELISA kit (R\&D systems Co., USA) (Tartaglia and Goeddel, 1992).

\section{- Coronary Efflux Lactate Dehydrogenase Levels: Coronary} efflux was collected during 30 minutes of reperfusion and was used for the measurement of lactate dehydrogenase (LDH) activity in IU/L spectrophotometrically (LDH UV Fluid, Rolf Greiner Biochemica, Germany).
LDH release was expressed per gram of tissue and was used as an index of myocardial injury (Astles et al., 1994).

\section{Statistical Analysis:}

Statistics were done using statistical package for the social sciences (SPSS) program (SPSS Inc., version 20). All data were expressed as mean \pm standard error of mean (SEM). Statistical significance for data was determined using a one-way analysis of variance (ANOVA) with posthoc test, LSD (least significant difference) and student' $s$ " $t$ " test for paired data. The level of significance was accepted when $\mathrm{P}$ $\leq 0.05$ (Armitage and Berry, 1987).

\section{RESULTS}

\section{Arterial Blood Pressure Changes (Table}

1): Concerning SBP and MAP, nontreated hyperthyroid (IR) rats showed significant elevation of these parameters compared to control rats $(\mathrm{P}<0.001)$. Moreover, the hyperthyroid (IR)-OCT rats exhibited significant reduction in their
SBP and MAP compared to non-treated hyperthyroid (IR) rats $(\mathrm{P}<0.001)$ and being none significantly changed compared to control group. The DBP did not show any significant difference among the three studied groups.

Table (1): Mean \pm SEM values of systolic blood pressure (SBP), diastolic blood pressure (DBP), and mean arterial pressure (MAP) in the three studied groups

\begin{tabular}{|c|c|c|c|}
\hline Groups & $\begin{array}{c}\text { Control (IR) } \\
\text { group } \\
(\mathbf{8})\end{array}$ & $\begin{array}{c}\text { Hyperthyroid } \\
\text { (IR) group } \\
(\mathbf{8})\end{array}$ & $\begin{array}{c}\text { Hyperthyroid (IR)- } \\
\text { OCT group } \\
(\mathbf{8})\end{array}$ \\
\hline $\mathbf{S B P}(\mathrm{mmHg})$ & $124 \pm 4.18$ & $161 \pm 6.50^{\mathbf{a}}$ & $120 \pm 3.71^{\mathbf{b}}$ \\
\hline $\mathbf{D B P}(\mathrm{mmHg})$ & $74 \pm 2.44$ & $72 \pm 1.55$ & $77 \pm 2.60$ \\
\hline $\mathbf{M A P}(\mathrm{mmHg})$ & $100 \pm 3.84$ & $127 \pm 2.98^{\mathbf{a}}$ & $103 \pm 4.33^{\mathbf{b}}$ \\
\hline
\end{tabular}

a: Significance from control group (IR) calculated by LSD.

b: Significance from hyperthyroid (IR) group calculated by LSD.

The number of observations was given in parentheses. 


\section{WESSAM EZZAT MORSY}

Body Weight (B.W) and Body Weight Gain (Table 2): Initial BW was not significantly changed among all rat groups. The final BW in the control group was significantly increased compared to the initial values $(\mathrm{P}<0.01)$, while the final $\mathrm{BW}$ in both hyperthyroid groups did not significantly change compared to their initial values. On the other hand, final BW and $\mathrm{BW}$ gain significantly reduced in both the hyperthyroid (IR) and hyperthyroid (IR)-OCT groups compared to control group ( $\mathrm{P}<0.01$ for both).

Table (2): Mean \pm SEM values of initial body weight (g), final body weight (g) and body weight gain $(\%)$ in the three studied groups

\begin{tabular}{|c|c|c|c|}
\hline Groups & $\begin{array}{c}\text { Control (IR) } \\
\text { group } \\
\text { Parameters }\end{array}$ & $\begin{array}{c}\text { Hyperthyroid } \\
\text { (IR) group } \\
(\mathbf{8})\end{array}$ & $\begin{array}{c}\text { Hyperthyroid (IR)- } \\
\text { OCT group } \\
(\mathbf{8})\end{array}$ \\
\hline $\begin{array}{c}\text { Initial body weight } \\
(\mathrm{g})\end{array}$ & $142.50 \pm 5.74$ & $154.25 \pm 6.11$ & $150 \pm 2.67$ \\
\hline $\begin{array}{c}\text { Final body weight } \\
(\mathrm{g})\end{array}$ & $167.50 \pm 3.89 *$ & $139.12 \pm 4.37 \mathrm{a}$ & $141.87 \pm 6.74 \mathrm{a}$ \\
\hline $\begin{array}{c}\text { Body weight gain } \\
(\%)\end{array}$ & $18.87 \pm 4.59$ & $8.62 \pm 4.94 \mathrm{a}$ & $5 \pm 4.84 \mathrm{a}$ \\
\hline
\end{tabular}

*: Significance from initial values calculated by Student`s t- test for paired data.

a: Significance from control group (IR) calculated by LSD.

The number of observations was given in parentheses.

Chronotropic activity (Table 3): The present study elucidated that non treated hyperthyroid rats showed significant tachycardia as those rats exhibited significant increase in baseline preischemia $\mathrm{HR}$ values compared to the euthyroid control rats $(\mathrm{P}<0.001)$.

After ischemia, a significant bradycardic response was evident during at all reperfusion values compared to their pre-ischemic values $(\mathrm{P}<0.001$ for all, except in hyperthyroid (IR) group at 5 minutes reperfusion $(\mathrm{P}<0.01)$. Moreover, the degree of bradycardia $(\Delta \mathrm{HR})$ after ischemia reperfusion was significantly higher in non-treated hyperthyroid (IR) group compared to euthyroid control group $(\mathrm{P}<0.001)$. Also, the percentage change in $\mathrm{HR}$ observed in non-treated hyperthyroid (IR) rats was significantly higher compared to control rats $(70 \pm 1.95$ versus $57 \pm 1.79, \mathrm{P}<0.001)$.

However, the baseline pre-ischemic HR values significantly decreased in the octreotide treated hyperthyroid group compared to the non-treated hyperthyroid group $(\mathrm{P}<0.001)$. A significant decrease in $\mathrm{HR}$ at all reperfusion values compared to their initial pre-ischemic value $(\mathrm{P}<0.001)$, with significantly reduced degree of bradycardia as evident by the lowest $\Delta \mathrm{HR}$ in this group. The percentage of HR change significantly reduced in the hyperthyroid (IR)-OCT rats compared to non-treated hyperthyroid (IR) rats $(55 \pm 1.33$ versus $70 \pm 1.95 \quad \mathrm{P}<0.001)$ (Figure 1). 
Table (3): Mean \pm SEM values of baseline heart rate (bpm) and effect of 30 minutes of ischemia-reperfusion of isolated hearts from the three studied groups

\begin{tabular}{|c|c|c|c|c|c|}
\hline Parameters & & & Reper & sion & \\
\hline Groups & $\begin{array}{l}\text { value } \\
\text { (bpm) }\end{array}$ & $\begin{array}{l}5 \text { min } \\
(b p m)\end{array}$ & $\begin{array}{l}15 \text { min } \\
(\mathrm{bpm})\end{array}$ & $\begin{array}{l}30 \text { min } \\
(\mathrm{bpm})\end{array}$ & $\begin{array}{c}\Delta \text { Change } \\
\text { (bpm) }\end{array}$ \\
\hline $\begin{array}{l}\text { Control (IR) } \\
\text { group }\end{array}$ & $229 \pm 3.54$ & $126 \pm 5.24 *$ & $103 \pm 2.6^{*}$ & $98 \pm 5^{*}$ & $131 \pm 3.83$ \\
\hline $\begin{array}{l}\text { Hyperthyroid } \\
\text { (IR) group }\end{array}$ & $276 \pm 7.36^{\mathrm{a}}$ & $203 \pm 7.06^{\mathbf{a}} *$ & $172 \pm 7.58^{\mathrm{a}} *$ & $82 \pm 3.66^{\mathbf{a}} *$ & $194 \pm 10.03^{\mathrm{a}}$ \\
\hline $\begin{array}{l}\text { Hyperthyroid } \\
\text { (IR)-OCT } \\
\text { group }\end{array}$ & $235 \pm 3.83^{\mathbf{b}}$ & $175 \pm 5.65^{\mathrm{ab}} *$ & $170 \pm 3.97^{\mathrm{a}} *$ & $105 \pm 2.94^{\mathbf{b}} *$ & $130 \pm 4.39^{b}$ \\
\hline
\end{tabular}

*: Significance from pre-ischemia baseline value calculated by Student`s t- test for paired data . a: Significance from control (IR) group, calculated by LSD.

b: Significance from hyperthyroid (IR), calculated by LSD.

Inotropic activity (Table 4): Results of inotropic parameters showed significant increase in pre-ischemic baseline PT, $\mathrm{PT} / \mathrm{LV}$ values $(\mathrm{P}<0.01)$ as well as significant prolongation in baseline TPT values $\quad(\mathrm{P}<0.001) \quad$ in $\quad$ non-treated hypethyroid rats compared to their corresponding values in euthyroid control rats).

After ischemia, non-treated hyperthyroid (IR) group exhibited a significant reduction of $\mathrm{PT}$ and $\mathrm{PT} / \mathrm{LV}$, prolongation in TPT as well as $1 / 2 \mathrm{RT}$ at 15 minute reperfusion values compared to their initial baseline pre-ischemic values $(\mathrm{P}<0.05, \quad \mathrm{P}<0.001 \quad$ and $\quad \mathrm{P}<0.01$ respectively). The same findings were also found at 30 minute reperfusion values $(\mathrm{P}<0.001$ for all). Moreover, $1 / 2$ RT of nontreated hyperthyroid rats didn't show any significant difference either in their baseline values or all post-ischemic reperfusion values compared to euthyroid control rats. The deteriorated inotropic recovery of hyperthyroid rats was also evident by the significantly increased $\Delta$ $\mathrm{PT}$ and $\Delta \mathrm{PT} / \mathrm{LV}(\mathrm{P}<0.01)$ and percentage change in PT and PT/LV (74.16 \pm 2.8$)$ compared to euthyroid control group $(59 \pm 7.01, \mathrm{P}<0.05)$.
On the other hand, the octreotide treated hyperthyroid rats showed improvement of their baseline inotropic parameters (PT, PT/LV and TPT) compared to non-treated group as demonstrated by the significant reduction in PT, PT/LV and TPT $(\mathrm{P}<0.01$ for all $)$.

After ischemia, the octreotide - treated hyperthyroid group revealed significant reduction in $\mathrm{PT}$ and $\mathrm{PT} / \mathrm{LV} \quad(\mathrm{P}<0.01$, $\mathrm{P}<0.01$ and $\mathrm{P}<0.001$ respectively) accompanied by a significant prolongation in $1 / 2$ RT $(<0.001, \mathrm{P}<0.01$ and $\mathrm{P}<0.001$ respectively) at all recorded reperfusion values and a significant prolongation of TPT $(\mathrm{P}<0.05$ and $\mathrm{P}<0.001)$ only at 15 and 30 minute reperfusion values compared to their initial baseline pre-ischemic values. Moreover, a significant low $\Delta$ change of PT and PT/LV parameters was observed in octreotide - treated hyperthyroid rats compared to non-treated hyperthyroid group $(\mathrm{P}<0.05)$. In addition, the percentage change in TPT significantly reduced in octreotide - treated hyperthyroid rats $(55.9 \pm 8.8)$ compared to non-treated hyperthyroid rats, and being non-significant from the euthyroid controls $(84.7 \pm 10.9, \mathrm{P}<0.05)$ (Figure 1). 


\section{WESSAM EZZAT MORSY}

Table (4): Mean \pm SEM of baseline values of Peak developed tension (PT, g), PT per left ventricular weight $(\mathrm{PT} / \mathrm{LV}, \mathrm{g} / 100 \mathrm{mg})$, half relaxation time $(1 / 2 \mathrm{RT}$, msec) and time to peak tension (TPT, msec) and effect of 30 minutes ischemia-reperfusion of hearts isolated from the three studied groups

\begin{tabular}{|c|c|c|c|c|c|c|}
\hline \multirow{2}{*}{\multicolumn{2}{|c|}{$\begin{array}{c}\text { Parameters } \\
\text { Groups }\end{array}$}} & \multirow{3}{*}{$\begin{array}{c}\begin{array}{c}\text { Baseline } \\
\text { value }\end{array} \\
6.14 \pm 0.36\end{array}$} & \multicolumn{3}{|c|}{ Reperfusion } & \multirow{3}{*}{$\begin{array}{c}\Delta \text { Change } \\
3.67 \pm 0.51\end{array}$} \\
\hline & & & \multirow{2}{*}{$\frac{5 \text { min }}{4.98 \pm 0.47 *}$} & \multirow{2}{*}{$\begin{array}{c}15 \mathrm{~min} \\
3.85 \pm 0.24 *\end{array}$} & \multirow{2}{*}{$\begin{array}{c}30 \text { min } \\
2.46 \pm 0.339 *\end{array}$} & \\
\hline \multirow{4}{*}{$\begin{array}{l}\text { Control (IR) } \\
\text { group }\end{array}$} & PT (g) & & & & & \\
\hline & $\begin{array}{l}\text { PT/LV } \\
\text { (g/100 } \\
\text { mg) }\end{array}$ & $1.59 \pm 0.09$ & $1.16 \pm 0.08 *$ & $0.71 \pm 0.1 *$ & $0.64 \pm 0.1 *$ & $0.95 \pm 0.13$ \\
\hline & $\begin{array}{c}\text { TPT } \\
(\mathrm{msec})\end{array}$ & $47.06 \pm 1.57$ & $58.97 \pm 1.84 *$ & $67.92 \pm 3.6^{*}$ & $74.98 \pm 4.19 *$ & $27.92 \pm 3.95$ \\
\hline & $\begin{array}{l}1 / 2 \mathrm{RT} \\
\text { (msec) }\end{array}$ & $71.76 \pm 3.45$ & $77.6 \pm 3.19$ & $89.32 \pm 2.54 *$ & $108.52 \pm 3.25 *$ & $36.76 \pm 3.2$ \\
\hline \multirow{4}{*}{$\begin{array}{l}\text { Hyperthyroid } \\
\text { (IR) group }\end{array}$} & PT (g) & $8.58 \pm 0.67^{\mathrm{a}}$ & $7.55 \pm 0.85^{\mathrm{a}}$ & $5.54 \pm 0.93^{\mathrm{a} *}$ & $2.16 \pm 0.23 *$ & $6.42 \pm 0.58^{a}$ \\
\hline & $\begin{array}{c}\mathrm{PT} / \mathrm{LV} \\
(\mathrm{g} / 100 \\
\mathrm{mg})\end{array}$ & $2.09 \pm 0.16^{\mathrm{a}}$ & $1.71 \pm 0.17^{\mathrm{a}}$ & $0.91 \pm 0.09 *$ & $0.53 \pm 0.05^{*}$ & $1.56 \pm 0.14^{\mathrm{a}}$ \\
\hline & $\begin{array}{c}\text { TPT } \\
(\text { msec) }\end{array}$ & $37.38 \pm 1.66^{\mathbf{a}}$ & $40.9 \pm 1.69^{\mathrm{a}}$ & $53.98 \pm 3.39^{a} *$ & $68.68 \pm 4.4 *$ & $31.31 \pm 3.89$ \\
\hline & $\begin{array}{l}1 / 2 \mathbf{R T} \\
(\mathrm{msec})\end{array}$ & $70.58 \pm 2.23$ & $72.56 \pm 2.53$ & $92.75 \pm 5.21 *$ & $107.06 \pm 2.97 *$ & $36.48 \pm 3.96$ \\
\hline \multirow{4}{*}{$\begin{array}{c}\text { Hyperthyroid } \\
\text { (IR) - OCT } \\
\text { group }\end{array}$} & $\mathbf{P T}(\mathbf{g})$ & $6.51 \pm 0.29^{\mathbf{b}}$ & $4.52 \pm 0.28^{\mathbf{b} *}$ & $4.51 \pm 0.26^{*}$ & $2.04 \pm 0.19 *$ & $4.48 \pm 0.29^{b}$ \\
\hline & $\begin{array}{c}\text { PT /LV } \\
\text { (g/100 } \\
\text { mg) }\end{array}$ & $1.59 \pm 0.08^{\mathbf{b}}$ & $1.1 \pm 0.12^{\mathbf{b} *}$ & $0.75 \pm 0.09 *$ & $0.5 \pm 0.05^{*}$ & $1.09 \pm 0.08^{b}$ \\
\hline & $\begin{array}{c}\text { TPT } \\
(\text { msec) }\end{array}$ & $45.06 \pm 1.46^{\mathrm{a}}$ & $46.81 \pm 1.38^{\mathbf{a b}}$ & $55.69 \pm 2.68^{\mathrm{a} *}$ & $69.94 \pm 3.87 *$ & $24.87 \pm 3.75^{b}$ \\
\hline & $\begin{array}{l}1 / 2 \text { RT } \\
(\text { msec) }\end{array}$ & $68 \pm 2.26$ & $91.12 \pm 4.32^{\mathrm{ab} *}$ & $92.75 \pm 5.21 *$ & $103.13 \pm 2.48 *$ & $35.13 \pm 2.39$ \\
\hline
\end{tabular}

*: Significance from pre-ischemia baseline value calculated by Student`s t- test for paired. a: Significance from control (IR) group, calculated by LSD data.

b: Significance from hyperthyroid (IR), calculated by LSD.

Myocardial flow rate (Table 5): The baseline values of MFR and MFR/LV and all values of reperfusion showed nonsignificant differences in the non-treated hyperthyroid (IR) group compared to control group. However, the octreotide treated group revealed significant increase in MFR and MFR/LV at 30 minute reperfusion value compared to non-treated hyperthyroid (IR) group $(\mathrm{P}<0.05)$.

All groups demonstrated nonsignificant change in the MFR and $\mathrm{MFR} / \mathrm{LV}$ at 5 minute reperfusion and a significant reduction at 30 minute reperfusion compared to their respective 
pre-ischemia baseline values $(\mathrm{P}<0.01$ in control and hyperthyroid (IR)-OCT groups and $\mathrm{P}<0.001$ in non-treated hyperthyroid (IR) group). Moreover, the changes of MFR and MFR/LV from baseline to post-ischemic values $(\triangle \mathrm{MFR}$
\& $\triangle \mathrm{MFR} / \mathrm{LV}$ ) as well as the percentage change of MFR and MFR/LV were nonsignificantly changed in both hyperthyroid groups $(46 \pm 1.2 \& 32 \pm 5.36$ respectively) compared to control group (36 \pm 7.19$)$ (Figure 1).

Table (5): Mean \pm SEM values of baseline myocardial flow rate (MFR, $\mathrm{ml} / \mathrm{min}$ ) and MFR per left ventricular weight $(\mathrm{ml} / \mathrm{min} / 100 \mathrm{mg})$, and effect of 30 minutes ischemia-reperfusion of hearts isolated from the three studied groups

\begin{tabular}{|c|c|c|c|c|c|c|}
\hline \multirow{2}{*}{\multicolumn{2}{|c|}{$\begin{array}{l}\text { Parameters } \\
\text { Groups }\end{array}$}} & \multirow{3}{*}{$\begin{array}{c}\begin{array}{c}\text { Baseline } \\
\text { value }\end{array} \\
5.11 \pm 0.43\end{array}$} & \multicolumn{3}{|c|}{ Reperfusion } & \multirow{3}{*}{$\begin{array}{c}\Delta \\
\text { Change } \\
1.9 \pm 0.44\end{array}$} \\
\hline & & & \multirow{2}{*}{$\frac{5 \mathbf{~ m i n}}{5.30 \pm 0.54}$} & \multirow{2}{*}{$\begin{array}{c}15 \mathrm{~min} \\
4.65 \pm 0.51\end{array}$} & \multirow{2}{*}{$\begin{array}{c}\text { 30min } \\
3.21 \pm 0.40 *\end{array}$} & \\
\hline Contro & MFR & & & & & \\
\hline group & $\begin{array}{l}\text { MFR /LV } \\
\text { mass }\end{array}$ & $1.32 \pm 0.1$ & $0.16 \pm 0.13$ & $0.9 \pm 0.09 *$ & $0.84 \pm 0.1 *$ & $0.48 \pm 0.1$ \\
\hline \multirow{2}{*}{$\begin{array}{r}\text { Hyperthyroid } \\
(\text { IR) group }\end{array}$} & MFR & $4.95 \pm 0.56$ & $4.87 \pm 0.64$ & $5.05 \pm 0.63$ & $2.71 \pm 0.33 *$ & $2.23 \pm 0.24$ \\
\hline & $\begin{array}{l}\text { MFR } / \mathbf{L V} \\
\quad \operatorname{mass}\end{array}$ & $1.21 \pm 0.13$ & $0.98 \pm 0.08$ & $0.81 \pm 0.1$ & $0.66 \pm 0.08 *$ & $0.55 \pm 0.05$ \\
\hline \multirow{2}{*}{$\begin{array}{c}\text { Hyperthyroid } \\
\text { (IR)-OCT } \\
\text { group }\end{array}$} & MFR & $6.03 \pm 0.46$ & $6.07 \pm 0.37$ & $4.18 \pm 0.31 *$ & $4.01 \pm 0.28^{\mathbf{b}} *$ & $2.01 \pm 0.47$ \\
\hline & $\begin{array}{c}\text { MFR /LV } \\
\text { mass }\end{array}$ & $1.47 \pm 0.11$ & $1.26 \pm 0.12$ & $1.22 \pm 0.1^{\mathbf{a ~ b}}$ & $0.98 \pm 0.06^{\mathbf{b}} *$ & $0.49 \pm 0.11$ \\
\hline
\end{tabular}

*: Significance from pre-ischemia baseline value calculated by Student`s t- test for paired data. a: Significance from control (IR) group, calculated by LSD.

b: Significance from hyperthyroid (IR), calculated by LSD.

Figure (1): Percentage change value of heart rate (bpm), peak developed tension (PT, g), time to peak tension (TPT, msec), half relaxation time $(1 / 2 \mathrm{RT}$, msec) and myocardial flow rate (MFR, $\mathrm{ml} / \mathrm{min}$ ), following ischemia-reperfusion of hearts isolated from the three studied groups

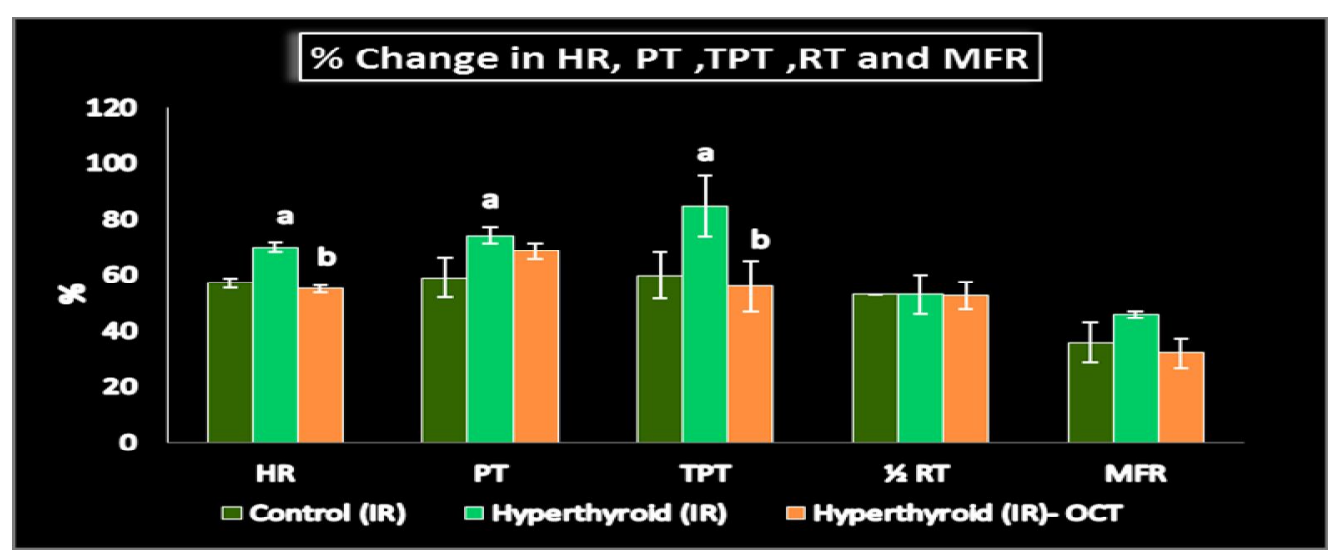

a: Significance from control (IR) group

b: Significance from hyperthyroid (IR) 


\section{WESSAM EZZAT MORSY}

Absolute Cardiac Weights and Cardiac Indices (Table 6): All rat groups exhibited non-significant changes regarding their whole heart (WH) while $\mathrm{WH} / \mathrm{BW}$ in both hyperthyroid groups showed significant increase compared to the control group ( $\mathrm{P}<0.001$ for both). The octreotide treated hyperthyroid (IR) group revealed no significant change compared to nontreated hyperthyroid (IR) group.
Regarding the absolute weights of left ventricle (LV) and cardiac index (LV/BW), they were significantly higher in both the hyperthyroid (IR) and hyperthyroid (IR)-OCT groups compared to control group $(\mathrm{P}<0.01 \quad \& \mathrm{P}<0.05$ respectively). Meanwhile, the hyperthyroid (IR)-OCT group showed no significant change compared to nontreated hyperthyroid (IR) group.

Table (6): Mean \pm SEM values of body weight $(\mathrm{g})$, and absolute cardiac weights $(\mathrm{mg})$ and cardiac indices of whole heart $(\mathrm{WH})(\mathrm{mg} / \mathrm{g})$, left ventricle $(\mathrm{LV})(\mathrm{mg} / \mathrm{g})$ in the three studied groups

\begin{tabular}{|c|c|c|c|}
\hline $\begin{array}{c}\text { Groups } \\
\text { Parameters }\end{array}$ & $\begin{array}{c}\text { Control (IR) } \\
\text { group } \\
(\mathbf{8})\end{array}$ & $\begin{array}{c}\text { Hyperthyroid (IR) } \\
\text { group } \\
(\mathbf{8})\end{array}$ & $\begin{array}{c}\text { Hyperthyroid (IR)-OCT } \\
\text { group } \\
(\mathbf{8})\end{array}$ \\
\hline Body weight (g) & $167.50 \pm 3.89$ & $139.12 \pm 4.37^{\mathbf{a}}$ & $141.87 \pm 6.74^{\mathbf{a}}$ \\
\hline $\mathbf{W H}(\mathbf{m g})$ & $680.78 \pm 18.93$ & $660.82 \pm 16.99$ & $655.18 \pm 12.29$ \\
\hline WH/BW (mg/g) & $3.25 \pm 0.18$ & $4.78 \pm 0.21^{\mathbf{a}}$ & $4.70 \pm 0.28^{\mathbf{a}}$ \\
\hline $\mathbf{L V ~ ( m g ) ~}$ & $385.96 \pm 6.0$ & $410.0 \pm 3.71^{\mathbf{a}}$ & $409.82 \pm 3.2^{\mathbf{a}}$ \\
\hline $\mathbf{L V} / \mathbf{W H}(\mathbf{m g} / \mathbf{m g})$ & $0.57 \pm 0.02$ & $0.62 \pm 0.01^{\mathbf{a}}$ & $0.63 \pm 0.01^{\mathbf{a}}$ \\
\hline
\end{tabular}

a: Significance from control group (IR) calculated by LSD at $\mathrm{P}<0.05$.

The number of observations was given in parentheses

Plasma free T3, T4 and TSH levels (figure 2): Regarding the hormonal profile, plasma free T3 levels were significantly increased in the hyperthyroid groups; $(2.14 \pm 0.036 \& 2.15 \pm 0.034$ respectively) compared to the control group $(0.76 \pm 0.08, \mathrm{P}<0.01)$. Also, a significant increase was observed in plasma free T4 $(10.97 \pm 1.44 \&$ $9.27 \pm 0.59$ respectively) compared to the control group $(1.78 \pm 0.13 \mathrm{P}<0.001)$, accompanied by a significant decrease in plasma TSH level $(0.04 \pm 0.01$ and $0.05 \pm 0.01$ versus $0.08 \pm 0.01$, $\mathrm{P}<0.001)$. These findings confirmed the successful induction of the hyperthyroid state in these groups.

Post-ischemic tumor necrosis factor $\alpha(\mathrm{TNF} \alpha)$ and malondialdehyde (MDA) in cardiac tissue (figure $3 \mathrm{a} \& \mathrm{~b}$ ): The present study showed that both cardiac tissue MDA as well as TNF $\alpha$ were significantly higher in hyperthyroid (IR) group compared to control group $(19.72 \pm 1.04$ versus
$13.56 \pm 1.39$ and $12.03 \pm 0.70$ versus $7.84 \pm 0.68$, $\mathrm{P}<0.01 \& \mathrm{P}<0.001$ respectively). On the other hand, both parameters were significantly reduced in octerotide treated hyperthyroid (IR) group $(11.79 \pm 0.62 \& 8.28 \pm 0.52)$ compared to untreated hyperthyroid (IR) group $(19.72 \pm 1.04 \& 12.03 \pm 0.7$ respectively, $\quad \mathrm{P}<0.001)$ and non-significantly different from control group.

Post-ischemic coronary flux lactate dehydrogenase (LDH) (figure 3c): The hyperthyroid (IR) rat group revealed significant elevation in $\mathrm{LDH}$ level compared to control group $(28.21 \pm 1.43$ versus $22.65 \pm 1.23, \mathrm{P}<0.01)$. However, LDH level was significantly reduced in octerotide - treated hyperthyroid (IR) group compared to untreated hyperthyroid (IR) group (24 \pm 0.90 versus $28.21 \pm 1.43, \mathrm{P}<0.05)$ and non-significantly different from control group. 
Figure (2): Free plasma T3 (ng/dl), T4 $\mu \mathrm{g} / \mathrm{dl}$ and TSH ( $\mu \mathrm{IU} / \mathrm{ml}$ ) levels in the three studied groups.
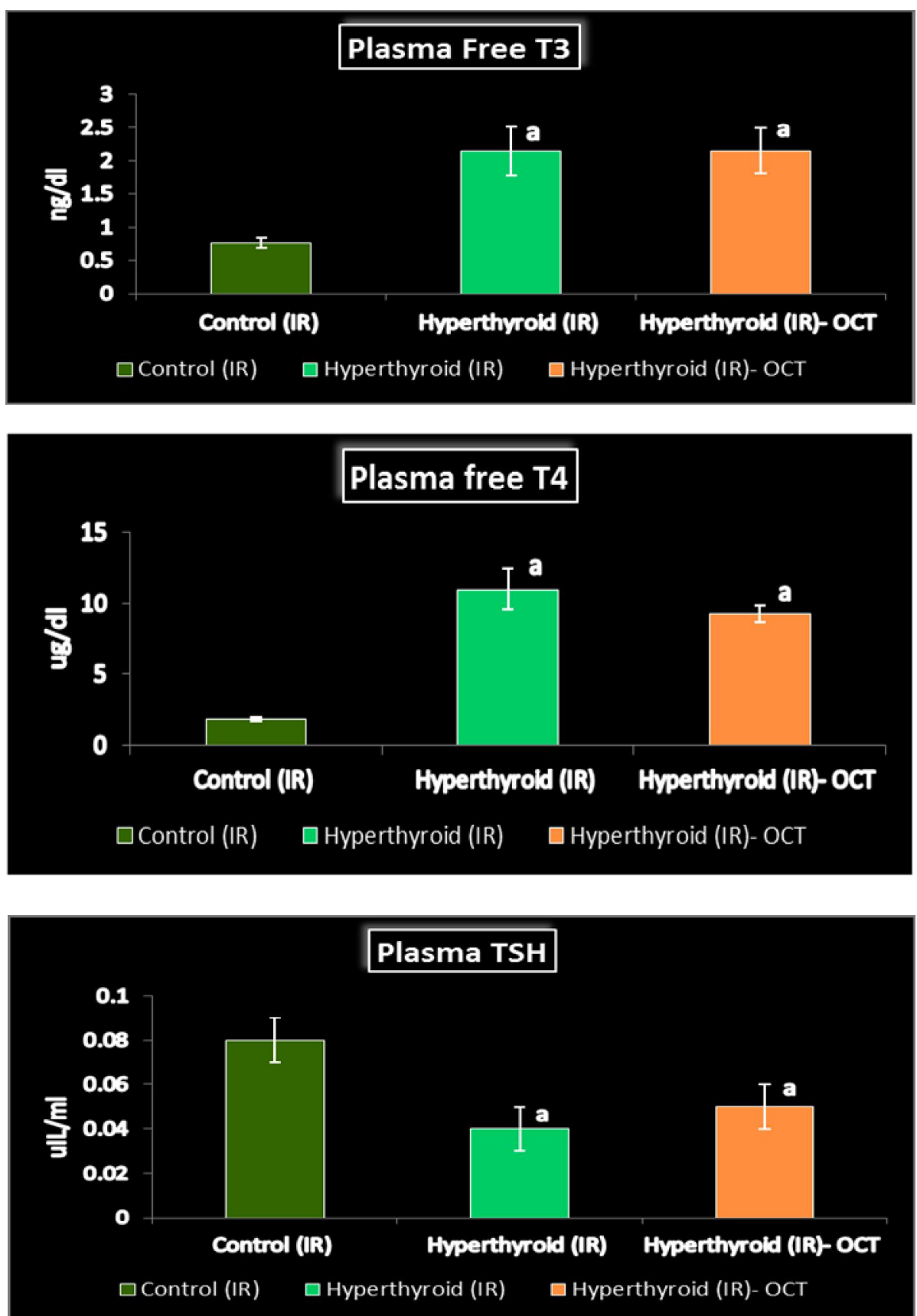

a: Significance from control group (IR) calculated by LSD. 
Figure (3): A) Cardiac tissue malondialdehyde (MDA, $\mu \mathrm{mol} / \mathrm{gm}$ ), B) tumor necrosis factor $\alpha(\mathrm{TNF} \alpha, \mathrm{pg} / \mathrm{ml})$ levels and C) lactate dehydrogenase (LDH, U/L) levels in coronary flux in the three studied groups
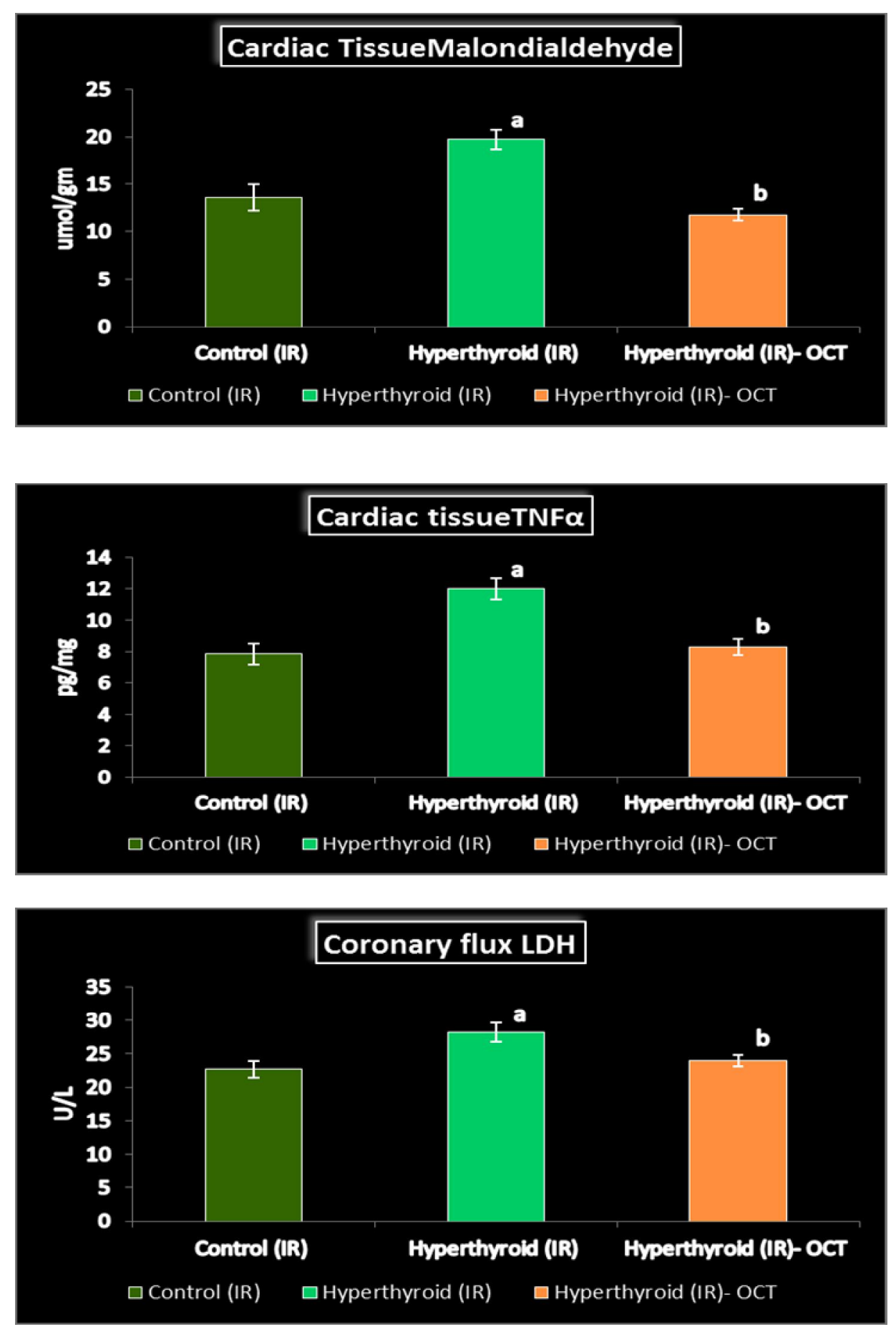

a: Significance from control group (IR) calculated by LSD.

b: Significance from hyperthyroid(IR)-OCT group calculated by LSD. 


\section{DISCUSSION}

The finding of the present study elucidated that final body weight, body weight gain were significantly decreased in rats rendered hyperthyroid. This could possibly be explained by increasing $\beta$ adrenoreceptor number on the membranes of adipocytes as result of excess throid hormones with up-regulation of the catecholamine - stimulated lipolysis (Obregon, 2008). Moreover, (Kim, 2008) observed increased energy expenditure and basal metabolic rate (BMR) in hyperthyroidism which could have added to the weight loss in the hyperthyroid groups.

The observed elevation in free plasma T3, T4 together with the subsequent decrease in TSH, in addition to reduced body weight in hyperthyroid groups confirmed the successful induction of the hyperthyroid state .

In fact, hyperthyroid rats in this study exhibited significantly increased $\mathrm{WH}$, $\mathrm{WH} / \mathrm{BW}, \mathrm{LV}$ and $\mathrm{LV} / \mathrm{WH}$ in comparison to euthyroid control rats, suggesting development of cardiac hypertrophy, in particular left ventricular hypertrophy. Myocardial hypertrophy could be a compensatory response to the increased load imposed by hypertension as evident by the significant elevation in SBP and MAP in non-treated hyperthyroid (IR) rats compared to euthyroid control rats

This cardiac growth in response to excess thyroid hormones involves cardiac myocyte enlargement and proliferation of fibroblasts and vascular cells (Kenessey and Ojamaa, 2006). Moreover, contractile and calcium-handling proteins are upregulated, resulting in a condition frequently referred as physiological cardiac hypertrophy (Thomas et al., 2005). The non-significant changes in initial baseline MFR and MFR/LV in nontreated hyperthyroid rats could further raise the assumption of compensated cardiac hypertrophy.

Unlike basal pre-ischemic coronary flow, ventricular performance was affected by excess thyroid hormones. Hearts from non-treated hyperthyroid rats displayed significant tachycardic response which was accompanied by enhanced ventricular contractility as evidenced by the significant increase in initial preischemic baseline values of $\mathrm{HR}, \mathrm{PT}$, $\mathrm{PT} / \mathrm{LV}$ and prolongation in TPT as well compared to their euthyroid controls. This observation was in agreement with previous reports (Venditti et al., 2002) which also indicated the higher susceptibility of the heart from hyperthyroid rats to ischemia-reperfusion injury .

The increase in chronotropism in hyperthyroid rats is probably caused by unbalanced sympatho-vagal tone with a relative adrenergic overdrive (Biondi et al., 2002). Moreover, (Thomas et al., 2005) ascribed the enhanced contractility and thereby ejection fraction to the ability of thyroid hormones to potentiate calcium-induced calcium release from sarcoplasmic reticulum during systolic period by up-regulation of ryanodine receptor expression levels and sarcoplasmic calcium ATP ase, as well as down-regulation of phospholambam expression levels that help calcium uptake by sarcoplasmic reticulum.

Thus, our findings together with the previous studies reinforce the notion that 


\section{WESSAM EZZAT MORSY}

the hyper-dynamic cardiovascular state in hyperthyroidism is in fact an adaptive response to the increase in the peripheral metabolic needs produced by excess thyroid hormones.

Isolated hearts in this study were subjected to zero-flow global ischemia followed by reperfusion. Results of isolated perfused hearts of non-treated hyperthyroid group revealed increased bradycardic responses to ischemiareperfusion (IR) as evidenced by significantly higher $\Delta \mathrm{HR}$ and percentage change in HR compared to euthyroid control group. Additionally, non-treated hyperthyroid hearts appear to be more influenced by IR injury and showed significantly deteriorated post-ischemic recovery of inotropic parameters (PT, PT/LV and TPT) and significantly higher $\Delta$ and $\%$ changes of these parameters compared to euthyroid controls .

Such results were consistent with Seara et al.(2018) who provided evidence that the pathophysiological progression of myocardial IR injury can be distinctly affected by levels of thyroid hormones and demonstrated that post-ischemic recovery of $\mathrm{LV}$ end-diastolic pressure (LVEDP), LVDP and dP/dt was impaired in thyrotoxic rat hearts.

This myocardial contractile dysfunction observed during reperfusion in hyperthyroidism was imputed to an increase in myocardial oxidative stress, which has been reported in the present study as cardiac tissue MDA levels were significantly elevated in non-treated hyperthyroid rats compared to euthyroid rats. Hyperthyroid state is characterized by an increase of oxygen consumption associated with higher electron flux in mitochondrial respiratory chain, a condition that may increase generation of ROS (Fernandez and Videla, 1993). Moreover, mitochondria are a possible site of ischemia-reperfusion damage. Actually, ischemia reduces in vitro mitochondrial respiration, an effect that is potentiated by reperfusion (Borutaite et al., 1995). Accordingly, Venditti et al. (2002) deduced that heart performance is strongly dependent on mitochondrial function and suggested that mitochondrial dysfunction during reoxygenation is due to an oxidative stress, which is more severe in hyperthyroid hearts.

Our results showed that, cardiac tissue $\mathrm{TNF} \alpha$ significantly increased in nontreated hyperthyroid rats, a finding similar to Al-Amran et al. ( 2013) who provided evidence that both ROS and TNF- $\alpha$ have deleterious effect on the myocytes and endothelial cells thus causing myocardial injury. Also, post-ischemic LDH level in coronary efflux of those rats were found to be significantly higher compared to euthyroid controls. Similar to our results, Shackebaei et al.(2012) has reported increased LDH levels and CK-MB in first 10 minutes of reperfusion which indicates damage to the heart during the IR period. This finding could contribute to higher susceptibility of the heart of hyperthyroid rats to IR.

Myocardial ischemia induces degranulation of resident mast cells and cleavage of membrane-bound TNF $\alpha$, both causes release of active TNF $\alpha$ in the ischemic myocardium to act in an autocrine, endocrine, and paracrine fashion (Gilles et al., 2003). Reperfusion of ischemic myocardium imposes an oxidant burden in which hydrogen 
peroxide activates P38 MAP kinase and contributes to ischemia reperfusioninduced TNF production (Al-Amran et al., 2013). Thus, the overwhelming production of pro-inflammatory mediators namely TNFa could further explain contractile dysfunction reported in nontreated hyperthyroid rats of our study.

Accumulating evidence strongly support the promise of octreotide preconditioning against hepatic ischemia reperfusion injury (Yang et al. 2014). In addition, it could reduce the edema and MDA in ischemic rat brain (Chen et al., 2012). Earlier findings of Wang et al. (2005) indicated that octreotide can mimic ischemic preconditioning to provide cardioprotection against ischemiareperfusion injury and to reduce infarct size in adult Wistar rats. But there is still no available data whether acute octreotide administration has a cardioprotective effect in ischemia-reperfusion model in conditions of diseased myocardium; in particular hyperthyroid states.

The results of the present study elucidated that hyperthyroid rats supplemented with octreotide, 20 minutes before scarification demonstrated significant improvement of the postischemic recovery of cardiac function compared to non-treated hyperthyroid rats. Interestingly, octreotide - treated hyperthyroid rats revealed significant decrease in the baseline tachycardia and the elevated developed peak tension and the prolonged TPT which was observed in non-treated hyperthyroid rats. Furthermore, it improved the chronotropic and some of inotropic parameters regarding their changes from baseline to post-ischemic values $(\Delta \mathrm{HR}, \Delta \mathrm{PT}$ and $\Delta$
PT/LV). The percent of decrease in HR following ischemia was significantly lower in octreotide treated rats (55\%) compared to non-treated hyperthyroid rats (70\%). Also, percent of TPT prolongation was significantly reduced in treated hyperthyroid rats $(55.9 \%)$ compared to $84.7 \%$ in non-treated hyperthyroid rats. Such effects of octreotide were associated with reduction of myocardial injury, as denoted by the significantly low coronary flux LDH level in treated hyperthyroid group compared to non-treated hyperthyroid group.

The aforementioned protective effects of octreotide could be due its antioxidant effect and/or its anti-inflammatory effect (determined by significantly low cardiac tissue MDA and TNF $\alpha$ respectively).

Consistently, Chen et al. (2012) reported the anti-oxidant and effects of octreotide which prevented oxidative damage by enhancing the antioxidant defenses superoxide dismutase and glutathione reductase, thereby; it reduced MDA; a product of lipid peroxidation that is always used to evaluate the severity of oxidative damage.

In support of our findings, Wang et al. (2015) demonstrated that octreotide may exert anti-inflammatory and neuroprotective effects on retinal injury induced by ischemia through inhibition of the activation of NF-kappa; an important redox-sensitive transcription factor that regulates the genes of many inflammatory mediators thus plays an important role in ischemic tissue damage .

Studies have shown that somatostatin analogues may influence leukocytic activities e.g infiltration, adhesion, and chemotaxis and also inhibit the generation 


\section{WESSAM EZZAT MORSY}

of ROS from leukocytes (Sener et al., 2005) and decrease the production of $\mathrm{TNF} \alpha$ and other inflammatory cytokines (IL1 $\beta$ and IL6) (Yang et al., 2013), suggesting that this drug might exert antiinflammatory properties.

Other mechanisms such as activation of protein kinase $\mathrm{C}$, mitochondrial ATPsensitive potassium channels (mitoKATP channels) and sarcolemmal KATP channels could be involved in octreotide induced cardioprotection. Opening of the mito KATP channel affects mitochondrial swelling and optimization of respiration, prevention of mitochondrial calcium overload and control of reactive oxygen radicals; all of which have possible linkages with cardioprotection during myocardial ischemia and infarction (O'Rourke, 2000 and Wang et al., 2005).

\section{CONCLUSION}

Hyperthyroid hearts are less tolerant to ischemia/reperfusion injury with higher susceptibility to oxidative stress as well as overproduction of pro-inflammatory mediators. Thus, pharmacological targets that defend oxidative challenge and confer anti-inflammatory properties, namely octreotide (somatotstatin analogue) are considered to have potential therapeutic effect and may protect against myocardial ischemic damage in hyperthyroidism. These effects inspire us to further explore how hyperthyroid patients with cardiovascular risks could benefit from long term octreotide administration.

\section{REFERENCES}

1. Al-Amran F.G, Hadi N.R and Al-Qassam H.S. (2013): Effects of Thyroid Hormone Analogue and a Leukotrienes Pathway-Blocker on Reperfusion Injury Attenuation after Heart
Transplantation. ISRN Pharmacology, 2013: 303717.

2. Armitage P. and Berry G. (1987): Statistical methods in medical research. 2nd edition; Blackwell Scientific publications, London. pp. 817-818.

3. Astles R., Wiklliams C.P. and Sedor F. (1994): Stability of plasma lactate in vitro in the presence of antiglycolytic agents. Clin Chem., 40: 1327-30.

4. Ayobe M.H. and Tarazi R.C. (1983): Beta receptors and contractile reserve in left ventricular hypertrophy. Hypertension, 5: 192197.

5. Biondi B., Palmieri E.A., Lombardi G. and Fazio S. (2002): Effects of Thyroid Hormone on Cardiac Function - The Relative Importance of Heart Rate, Loading Conditions, and Myocardial Contractility in the Regulation of Cardiac Performance in Human Hyperthyroidism .The Journal of Clinical Endocrinology \& Metabolism, 87(3):968-74.

6. Borutaite V., Mildaziene V., Brown G.C. and Brand M.D. (1995): Control and kinetic analysis of ischemia-damaged heart mitochondria. Which parts of the oxidative phosphorylation system are affected by modification of ischemia? Biochim Biophys Acta., 1272:154-158.

7. Chen L., Wang L. and Zhang X. (2012): "The protection by octreotide against experimental ischemic stroke: up-regulated transcription factor Nrf2, HO-1 and down-regulated NF-kB expression" Brain Research, 1475,.80-87.

8. Fernandez V. and Videla L.A. (1993): Influence of hyperthyroidism on superoxide radical and hydrogen peroxide production by rat liver submitochondrial particles. Free Radical Research Communications, 18: 329335 .

9. Fisher D.A. (1996): Physiological variations in thyroid hormones; physiological and pathophysiological considerations. Clinical Chemistry, 42: 135-159.

10. Gilles S., Zahler S., Welsch U., Sommerhoff C.P. and Becker B.F. (2003): Release of TNFalpha during myocardial reperfusion depends 
on oxidative stress and is prevented by mast cell stabilizers. Cardiovasc Res., 60 (3):608-16.

11. Jiang J.Y., Imai Y., Umezu M., and Sato E. (2001): Characteristics of infertility in female hypothyroid mice. Reproduction, 122: 695700.

12. Kenessey A. and Ojamaa K. (2006): Thyroid hormone stimulates protein synthesis in the cardiomyocyte by activating the Akt-mTOR and p70S6K pathways. J Biol Chem. American Society for Biochemistry and Molecular Biology, 281: 20666 \pm 20672 .

13. Kim B. (2008): Thyroid Hormone as a Determinant of Energy Expenditure and the Basal Metabolic Rate. Thyroid, 18(2):141-4.

14. Komeda K., Mizuma Y. and Oki Y. (1977): Effect of thyroid hormone on the genetically controlled activity of serum esterase in mice. Japan J. Genetics, 520:407-411.

15. Obregon M.J. (2008): Thyroid hormone and adipocyte differentiation. Thyroid, 18: 185-95.

16. Ohkawa H., Ohishi W. and Yagi K. (1979): Assay for lipid peroxides in animal tissues by thiobarbituric acid reaction. Anal Biochem., 95(2):351-358.

17. O'Rourke B. (2000): Myocardial KATP channels in preconditioning. Circ Res.; 87:845855.

18. Pantos C., Mourouzis I., Saranteas T., Brozou V., Galanopoulos G., and Kostopanagiotou G. (2011): Acute T3 treatment protects the heart against ischemiareperfusion injury via TR $\alpha 1$ receptor. Mol Cell Biochem. Springer US, 353: 235-241.

19. Rodondi N., Bauer D.C., Cappola A.R., Cornuz J., Robbins J. and Fried L.P. (2008): Subclinical Thyroid Dysfunction, Cardiac Function, and the Risk of Heart Failure. The Cardiovascular Health Study. J Am Coll Cardiol., 52: 1152 \pm 1159 .

20. Seara F.A.C., Maciel L., Barbosa R.A.Q, Rodrigues N.C., Silveira A.L.B., Marassi M.P., Carvalho A.B., Nascimento J.H.M. and Olivares E.L. (2018): Cardiac ischemia/reperfusion injury is inversely affected by thyroid hormones excess or deficiency in male Wistar rats. PLoS ONE, 13(1):e0190355.

21. Sener G., Setinel C., Erkanli G., Gedik N. and Ye?gen B.C. (2005): Octreotide ameliorates sepsis-induced pelvic inflammation in female rats by a neutrophil-dependent mechanism. Peptides, 26(3): 493-499.

22. Shackebaei D., Asadmobini A., Hesari M., Vaezi M. and Shahdi S. (2012): Cardioprotective effect of diazepam on ischemia-reperfused isolated hyperthyroid rat heart. Turk. J. Biol., 36:598-605.

23. Smith W.H., Nair R.U., Adamson D., Kearney M.T., Ball S.G. and Balmforth A.J. (2005): Somatostatin receptor subtype expression in the human heart: Differential expression by myocytes and fibroblasts. J. Endocrinol., 187:379-386.

24. Tartaglia L. and Goeddel D. (1992): Two TNF receptors. Immunol. Today, 13: 151-153.

25. Thomas T.A., Kuzman J.A., Anderson B.E., Andersen S.M.K., Schlenker E.H. and Holder M.S. (2005): Thyroid hormones induce unique and potentially beneficial changes in cardiac myocyte shape in hypertensive rats near heart failure. Am J Physiol Heart Circ Physiol., 288: $\mathrm{H} 2118 \pm 22$.

26. Venditti, P., Agnisola, C. and Di Meo, S. (2002): Effect of ischemia-reperfusion on heart mitochondria from hyperthyroid rats. Cardiovasc. Res., 56: 76-85.

27. Wang T.L, Huang Y.H and Chang H. (2005): Somatostatin analogue mimics acute ischemic preconditioning in a rat model of myocardial infarction. J Cardiovasc Pharmaco., 45(4):327-332.

28. Wang Y.Y., Jiao B., Guo W.G., Che H.L. and Yu Z.B. (2015): Excessive thyroxine enhances susceptibility to apoptosis and decreases contractility of cardiomyocytes. Mol. Cell. Endocrinol., 320: 67-75.

29. Widiapradja A., Chunduri P. and Levick S. (2017): The role of neuropeptides in adverse myocardial remodeling and heart failure Cell Mol Life Sci., 74(11): 2019-2038.

30. Yang J., Sun H., Takacs P., Zhang Y., Liu J., Chang Y. and Candiotti K.A. (2013): The 
effect of octreotide on hepatic ischemiareperfusion injury in a rabbit model. Transplant Proc., 45(6):2433-8.

31. Yang J., Sun H., Guan R., Liu W., Xia Y., Zhao J. and Liu J. (2014): Hepatocellular protein profiles after hepatic ischemia/reperfusion injury with or without octreotide preconditioning in a rabbit model. Transplant Proc., 46(10):3282-8.
32. Zaman J., Jeddi S. and Ghasemi A. (2014): The effects of ischemic postconditioning on myocardial function and nitric oxide metabolites following ischemia-reperfusion in hyperthyroid rats. Korean J. Physiol. Pharmacol., 18:481-487. 


\section{تأثير نظير السو ماتوستاتين (اكتربوتيد) على إعادة التروبة بعد الاقفار الدموى في الجرذان البيضاء البالغة المصابة بفرط نشاط الغدة الدرقبة$$
\text { وسام عزت مرسى }
$$

قسم الفسيولوجيا الطبيةـ كلية الطب- جامعة عين شمس

خلفية البحث : يرتبط اختلال وظيفة الغدة الدرقية بارتفاع معدل الامر اض القلبية. ويزيد فرط نشاط

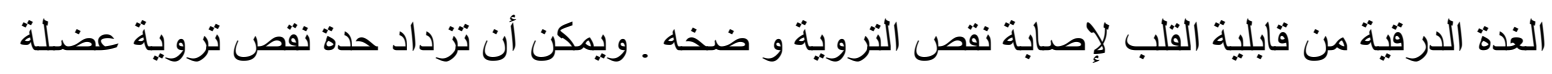

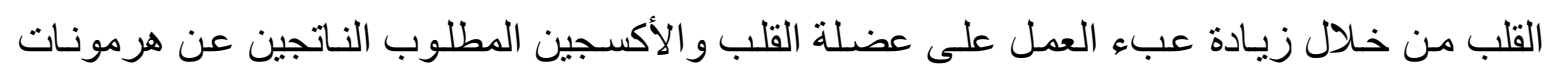
الغدة الدرقية الز ائدة.

الهـف من البحث: دراسـة إضطرب وظيفة القلب الناجم عن نقص الترويـة في الجرذان المصـابة

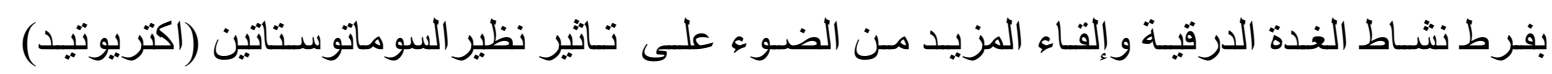
(CCT تروية الدم.

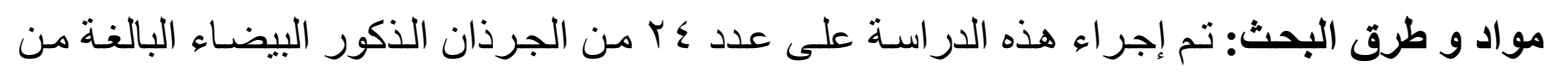

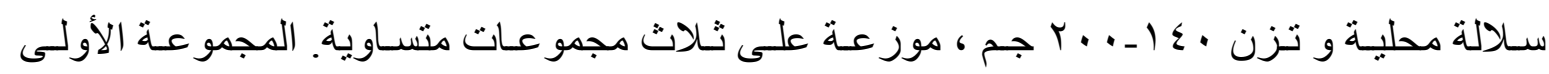

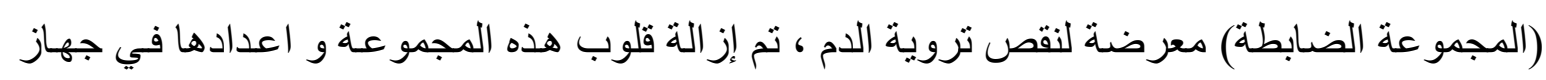
Langendorff

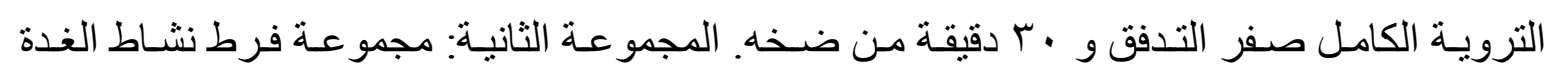

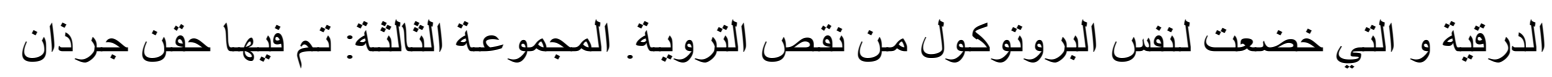

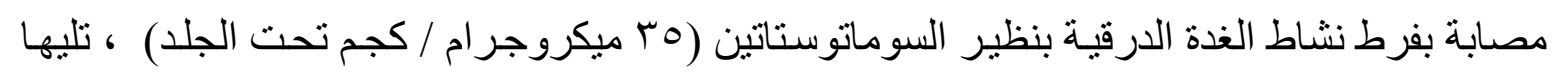

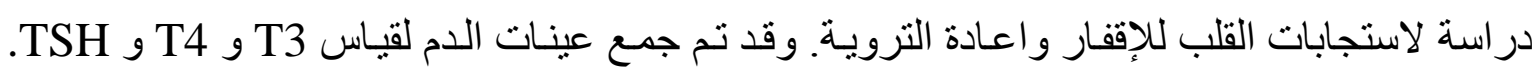

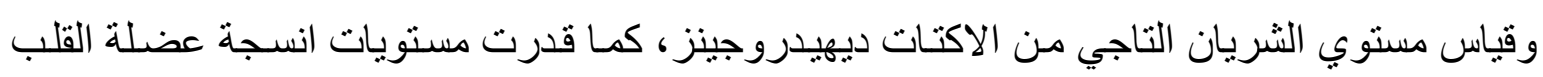
من المالوندايلدهيد ومعامل النخر ألفا.

النتائج: أظهرت جرذان فرط نشاط الغدة الدرقية ارتفاعًا ملحوظًا في مستويT3 و T4 في البلازمـا مع انخفاض مصاحب في مستويات TSH. وقد لوحظ انخفاض كبير في وزن الجسم و نسبة الزياده

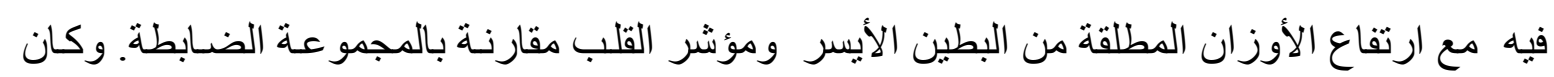




\section{WESSAM EZZAT MORSY}

الضغط الانقباضي ومتوسط ضغط الدم مرتفعين بشكل ملحوظ ، و أظهرت تللك الجرذان درجة أعلى بكثير من عدم انتظام دقات القلب و التغير في نسبة مئوية لهذا التغير ، وكذللك زيادة كبيرة في مؤشر وات

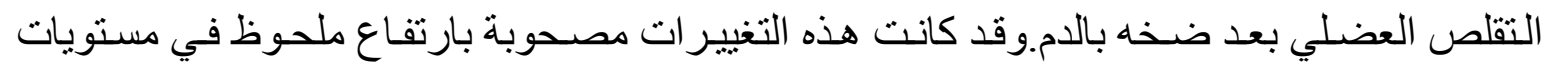
الثريان التاجي من الاكتات ديهيدروجينيز ونسيج القلب من المالوندايلدهيد ومعامل النخر ألفا. بينما أظهر إعطاء الأوكتريوتيد إلى جرذان فرط نشاط الغدة الدرقية تحسنًا في معايير ضربات

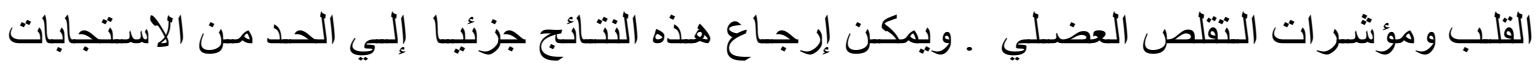

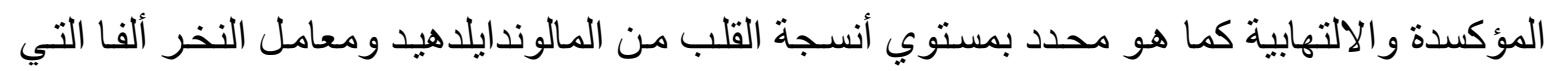
انخفضت بشكل كبير.

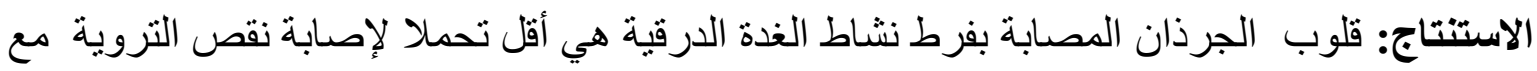

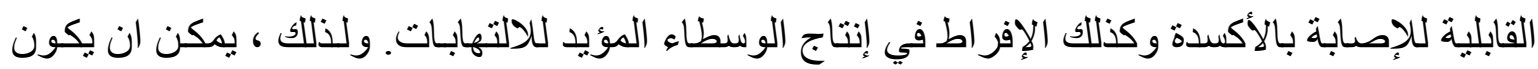

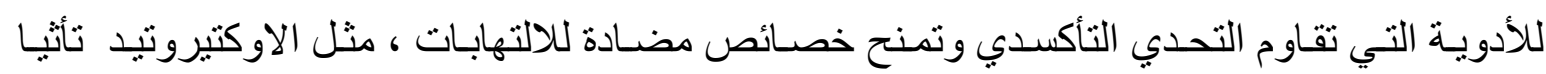
علاجيا محتملا ، وربما حماية ضد تلف عضلة القلب الناتج عن نقص الترويـة في حالات فرط نشـاط

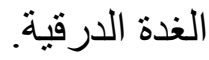

